\title{
Single Postulate Formulation of the Theory of Special Relativity
}

\author{
A. Sfarti \\ 387 Soda Hall, UC Berkeley, Berkeley, United States \\ Email: egas@pacbell.net
}

\begin{abstract}
In a prior paper [1] we have presented a novel derivation of the special theory of relativity (STR) using only one principle, the principle of relativity. In the current note we present a novel, different derivation that is more direct.
\end{abstract}

Keywords: Special theory of relativity, relativity principle

PACS: $03.30 .+\mathrm{p}$

\section{Introduction: Derivation of the Clock Synchronization Rule}

We start the new derivation by re-explaining the approach we took in our prior paper [1]. The setup comprises a rod $\mathrm{AB}$ with a light source at the $\mathrm{A}$ end and a mirror at the $\mathrm{B}$ end. The rod is at rest with respect to frame $\mathrm{k}$ of coordinates $(\xi, \psi, \zeta, \tau)$ and is moving with respect to frame $\mathrm{K}$ [2] of coordinates $(\mathrm{x}, \mathrm{y}, \mathrm{z}, \mathrm{t})$. Frames $\mathrm{k}$ and $\mathrm{K}$ have their axes aligned and there is a movement with constant velocity $\mathrm{v}$ along the $\mathrm{x}$-axis. Initially, the rod is oriented parallel with the commonly aligned $\mathrm{x}$ axis of $\mathrm{k}$ and $\mathrm{K}$. Later in the gedanken-experiment the rod is rotated perpendicular to the $\mathrm{x}$-axis, in a departure from Einstein's approach. As we will see, this departure will enable the derivation of the Lorentz transforms between the frames having only the principle of relativity as a starting point. In frame $\mathrm{k}$ the proper length of the rod is $\rho_{A B}$. A light signal starts at time $\tau_{A}$ from end A arriving at time $\tau_{B}$ at the other end $\mathrm{B}$ and reflects from end $\mathrm{B}$ arriving back to $\mathrm{A}$ at time $\tau_{A}^{\prime}$

$$
\tau_{B}-\tau_{A}=\tau_{A}^{\prime}-\tau_{B}=\frac{\rho_{A B}}{c}
$$

Frame K "sees" a different length $r_{A B}$ along the x-axis, a different time t and...a possibly different (yet isotropic) light speed c'. During the light trip from A to B the mirror at the B end of the rod recedes with a speed $v$ in frame $\mathrm{K}$ :

$$
t_{B}-t_{A}=\frac{r_{A B}}{c^{\prime}-v}
$$

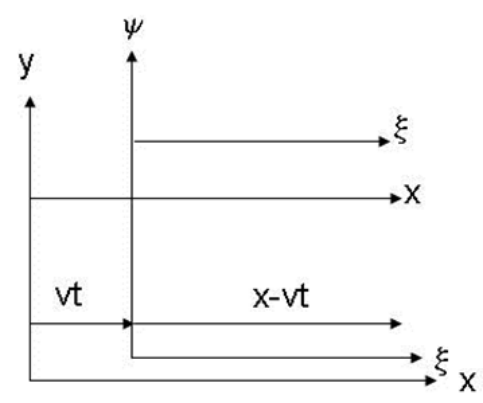

Figure 1. Two inertial frames of reference

During the light trip from B to A the mirror at the B end of the rod recedes with a speed $-\mathrm{v}$ : 


$$
t_{A}^{\prime}-t_{B}=\frac{r_{A B}}{c^{\prime}+v}
$$

It is sensible to assume that $r_{A B}=r_{B A}$, so

$$
\left(t^{\prime}{ }_{A}-t_{B}\right)\left(c^{\prime}+v\right)=\left(t_{B}-t_{A}\right)\left(c^{\prime}-v\right)
$$

This is the new, more general synchronization rule than ${ }^{2}$. In our gedanken-experiment we assume that the rod in frame $\mathrm{k}$ gets rotated $90^{\circ}$. For the new orientation, the length of the rod, measured in both $\mathrm{k}$ and $\mathrm{K}$ is $\rho_{A B}$. The whole setup becomes very similar with the setup of the Michelson Morley experiment. While the ray of light bounces from A to B and back, the observer in K "sees" it running the path seen in fig.2. Let's examine the four-space events experienced by the photons bouncing between the mirrors $\mathrm{A}$ and $\mathrm{B}$ in frames $\mathrm{k}$ and $\mathrm{K}$. Here is the list of events:

Event 0 [photon emitted at A ] $(0,0,0,0)$ in k corresponding to $(0,0,0,0)$ in $\mathrm{K}$

Event 1 [photon reflected at B], $\left(\rho_{A B}, 0,0, \rho_{A B}\right)$ in k vs. $\left(r_{A B} c^{\prime} /\left(c^{\prime}-v\right), 0,0, r_{A B} c^{\prime} /\left(c^{\prime}-v\right)\right)$ in $\mathrm{K}$

Event 2 [photon returns at $\mathrm{A}$ ] $\left(0,0,0,2 \rho_{A B}\right)$ in $\mathrm{k},\left(2 v c^{\prime} r_{A B} /\left(c^{\prime 2}-v^{2}\right), 0,0,2 c^{\prime 2} r_{A B} /\left(c^{\prime 2}-v^{2}\right)\right)$ in $\mathrm{K}$

Event 3 [reflection at rotated B] $\left(0, \rho_{A B}, 0, \rho_{A B}\right)$ vs. $\left(v \rho_{A B} / \sqrt{c^{\prime 2}-v^{2}}, \rho_{A B}, 0, \rho_{A B} c^{\prime} / \sqrt{c^{\prime 2}-v^{2}}\right)$ in $\mathrm{K}$

Event 4 [photon returns to A] $\left(0,0,0,2 \rho_{A B}\right)$ in k vs. $\left(2 v \rho_{A B} / \sqrt{c^{\prime 2}-v^{2}}, 0,0,2 \rho_{A B} c^{\prime} / \sqrt{c^{\prime 2}-v^{2}}\right)$ in $\mathrm{K}$

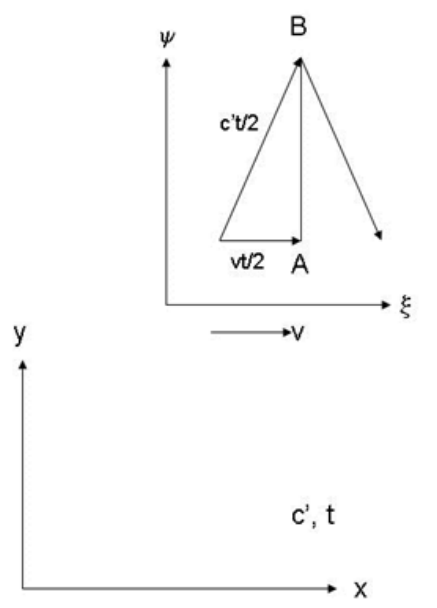

Figure 2. Modified setup

Since events 2 and 4 are identical in frame k, they are also identical in frame K. From this, we find out immediately that:

$$
r_{A B}=\rho_{A B} \sqrt{1-\frac{v^{2}}{c^{\prime 2}}}
$$

We are now ready to proceed with the derivation of the Lorentz transforms.

\section{Derivation of the Lorentz Transforms}

If we assume a linear transformation between $\mathrm{k}$ and $\mathrm{K}$ then:

$$
\left[\begin{array}{l}
x \\
c^{\prime} t
\end{array}\right]=\left[\begin{array}{ll}
A & B \\
C & D
\end{array}\right]\left[\begin{array}{l}
\xi \\
c \tau
\end{array}\right]
$$

From the data at event 2, we obtain: 


$$
\begin{gathered}
\frac{2 r_{A B} c^{\prime}}{c^{\prime 2}-v^{2}}=A^{*} 0+2 B \rho_{A B} \\
\frac{2 r_{A B} c^{\prime}}{c^{\prime 2}-v^{2}}=C^{*} 0+2 D \rho_{A B} \\
B=\frac{v / c^{\prime}}{\sqrt{1-\left(v / c^{\prime}\right)^{2}}} \\
D=\frac{1}{\sqrt{1-\left(v / c^{\prime}\right)^{2}}}
\end{gathered}
$$

In a similar manner, from the data at event 1 , we obtain:

$$
\begin{aligned}
& A=\frac{1}{\sqrt{1-\left(v / c^{\prime}\right)^{2}}} \\
& C=\frac{v / c^{\prime}}{\sqrt{1-\left(v / c^{\prime}\right)^{2}}}
\end{aligned}
$$

Introducing the notation $\mathrm{u}=\mathrm{c}^{\prime} \mathrm{t}, \mathrm{U}=\mathrm{c}$ the generalized Lorentz transformations are therefore:

$$
\begin{aligned}
& x(\xi, U)=\frac{1}{\sqrt{1-\frac{v^{2}}{c^{\prime 2}}}}\left(\xi+\frac{v}{c^{\prime}} U\right) \\
& u(\xi, U)=\frac{1}{\sqrt{1-\frac{v^{2}}{c^{\prime 2}}}}\left(U+\frac{v}{c^{\prime}} \xi\right)
\end{aligned}
$$

The equations can be algebraically inverted in order to obtain the inverse transforms:

$$
\begin{aligned}
& \xi(x, u)=\frac{1}{\sqrt{1-\frac{v^{2}}{c^{\prime 2}}}}\left(x-\frac{v}{c^{\prime}} u\right) \\
& U(x, u)=\frac{1}{\sqrt{1-\frac{v^{2}}{c^{\prime 2}}}}\left(u-\frac{v}{c^{\prime}} x\right)
\end{aligned}
$$

By using the principle of relativity in order to reverse the situation between frames $\mathrm{k}$ and $\mathrm{K}$ we derive a different set of transformations instead of (2.6):

$$
\begin{aligned}
& \xi(x, u)=\frac{1}{\sqrt{1-\frac{v^{2}}{c^{2}}}}\left(x-\frac{v}{c} u\right) \\
& U(x, u)=\frac{1}{\sqrt{1-\frac{v^{2}}{c^{2}}}}\left(u-\frac{v}{c} x\right)
\end{aligned}
$$

By comparing (12.6) with (2.7) we deduce immediately $c^{\prime}=c$. In other words, by using the generalized synchronization rule (1.4) $\mathrm{c}=\mathrm{c}^{\prime}$ (the principle of light speed constancy) becomes a consequence of the principle of relativity.

\section{Special Relativity vs. Quantum Gravity}

In the contemporary theories of Quantum Gravity [3], the locality of physics is realized in a new way, called relative locality. Observers using a coordinate system whose origin is adjacent to any interaction describe it as local, as in special relativity. But observers using coordinates whose origin is distant from that interaction are constrained to describe it by coordinates in which the event appears to be non-local. 
These non-localities, which can be created and eliminated by translations, are not real non-localities. They occur, not because of any subjectivity of reality, but because, as Einstein showed in his 1905 paper [2], the description of distant events by an observer is constructed by exchange of light signals. In the class of these Quantum Gravity theories this coordinatization becomes energy dependent and light speed in vacuum is no longer invariant. A series of experiments set to put limits on the energy effects on the light speed starting with [3] in 1999.

More recent experiments [4] using the binary neutron star merger, observed via gravitational waves, compared with the speed of many frequencies of EM, and gamma rays, have placed extremely stringent bounds on any such phenomenon of light speed dependency on energy. For all practical purposes the experiment rules out such effect. All the waves arrived at the same time to better than one part in $10^{15}$. Thus, Quantum Gravity is being put in total agreement with Special Relativity.

\section{Conclusions}

We have re-derived STR, from base principles, employing only the postulate of relativity.

\section{References}

1. A. Sfarti "Generalization of Einstein's synchronization for the case of anisotropic light speed", Can. J. Phys., 87(9): p.969, (2009).

2. A. Einstein, "On the Electrodynamics of Moving Bodies", Annalen der Physik 17, p.891 (1905).

3. S. D. Biller et al., "Limits to Quantum Gravity Effects on Energy Dependence of the Speed of Light from Observations of TeV Flares in Active Galaxies", Phys. Rev. Lett. 83, 2108, (1999).

4. B. P. Abbott et al., "Gravitational Waves and Gamma-Rays from a Binary Neutron Star Merger: GW170817 and GRB 170817A", The Astrophysical Journal Letters, 848:L13, (2017). 